\title{
Random forcing of geostrophic motion in rotating stratified turbulence $\odot$ ( $)$
}

Cite as: Phys. Fluids 29, 126602 (2017); https://doi.org/10.1063/1.5004986

Submitted: 15 September 2017 • Accepted: 05 December 2017 • Published Online: 29 December 2017

Michael L. Waite

\section{COLLECTIONS}

F This paper was selected as Featured

SCI This paper was selected as Scilight

\section{ARTICLES YOU MAY BE INTERESTED IN}

Inertial Ranges in Two-Dimensional Turbulence

The Physics of Fluids 10, 1417 (1967); https://doi.org/10.1063/1.1762301

Random forcing of three-dimensional homogeneous turbulence

Physics of Fluids 11, 1880 (1999); https://doi.org/10.1063/1.870050

Transfer of energy to two-dimensional large scales in forced, rotating three-dimensional turbulence

Physics of Fluids 11, 1608 (1999); https://doi.org/10.1063/1.870022

\section{Physics of Fluids}




\title{
Random forcing of geostrophic motion in rotating stratified turbulence
}

\author{
Michael L. Waite ${ }^{\mathrm{a})}$ \\ Department of Applied Mathematics, University of Waterloo, 200 University Avenue West, Waterloo, Ontario \\ N2L 3G1, Canada
}

(Received 15 September 2017; accepted 5 December 2017; published online 29 December 2017)

\begin{abstract}
Random forcing of geostrophic motion is a common approach in idealized simulations of rotating stratified turbulence. Such forcing represents the injection of energy into large-scale balanced motion, and the resulting breakdown of quasi-geostrophic turbulence into inertia-gravity waves and stratified turbulence can shed light on the turbulent cascade processes of the atmospheric mesoscale. White noise forcing is commonly employed, which excites all frequencies equally, including frequencies much higher than the natural frequencies of large-scale vortices. In this paper, the effects of these high frequencies in the forcing are investigated. Geostrophic motion is randomly forced with red noise over a range of decorrelation time scales $\tau$, from a few time steps to twice the large-scale vortex time scale. It is found that short $\tau$ (i.e., nearly white noise) results in about $46 \%$ more gravity wave energy than longer $\tau$, despite the fact that waves are not directly forced. We argue that this effect is due to wave-vortex interactions, through which the high frequencies in the forcing are able to excite waves at their natural frequencies. It is concluded that white noise forcing should be avoided, even if it is only applied to the geostrophic motion, when a careful investigation of spontaneous wave generation is needed. Published by AIP Publishing. https://doi.org/10.1063/1.5004986
\end{abstract}

\section{INTRODUCTION}

In idealized numerical simulations of turbulence, random forcing is often employed to represent the injection of kinetic energy at large scales or the transfer of energy from scales larger than the domain. Such forcing is convenient because the length scale and other characteristics can be controlled, e.g., by selectively forcing particular Fourier modes. Forcing is usually random in time, with white noise forcing being a common approach. ${ }^{1-3}$ Other non-random approaches are based on fixing the energy in a particular wavenumber band, ${ }^{4}$ fixing the energy injection rate, ${ }^{5,6}$ or relaxation to a prescribed large-scale flow. ${ }^{7}$ The underlying assumption in all studies that use idealized forcing, which is rarely verified, is that the details of the resulting turbulence do not depend too much on the forcing parameters. ${ }^{8}$ As a result, such forcing is a useful tool for exploring generic properties of turbulent flows.

White noise forcing is natural for homogeneous isotropic turbulence because it is unbiased in time. The forcing variance determines the energy injection rate (on average, or precisely if forcing-velocity correlations are removed ${ }^{1}$ ). All resolved time scales are excited equally. However, in geophysical flows, density stratification and system rotation introduce additional natural time scales and modes of motion, and the choice of forcing becomes more involved. Depending on the problem, it may or may not make sense to force all of these modes and/or frequencies. Rotating stratified flows can be decomposed into slow geostrophic motion and fast inertiagravity waves (IGWs), e.g., via the linear normal modes of

a)Electronic mail: mwaite@uwaterloo.ca the Boussinesq equations. ${ }^{9}$ Studies of forced rotating stratified turbulence generally fall in one of three categories: (i) force all fields randomly, ${ }^{2,10-12}$ thereby exciting both geostrophic motion and waves at the forcing scale; or selectively force only the (ii) slow geostrophic motion, ${ }^{3,13-15}$ or (iii) fast wave motion. ${ }^{16}$ Approach (i) is simple, but approach (ii) more closely represents the dynamics of the atmosphere and ocean away from wave sources, since large-scale motion is dominated by quasi-geostrophic flow. In all cases, the forcing is usually white noise or red noise correlated over a short time scale.

Forced rotating stratified turbulence can be used to investigate the downscale energy cascade through the atmospheric mesoscale. ${ }^{3,14,15}$ In such studies, approach (ii) must be taken. This idealized setup is capable of reproducing the general shape of the observed kinetic energy (KE) spectrum, which has a -3 spectral slope at large scales followed by a shallower $-5 / 3$ slope in the mesoscale. ${ }^{17}$ At large scales, energy is dominated by geostrophic motion, which has an approximately -3 spectral slope. The wave energy spectrum is shallower but lower in amplitude. Because of the different spectral slopes, the geostrophic and wave energy spectra cross downscale, below which both follow an approximately $-5 / 3$ slope. ${ }^{3,15}$ Even though large-scale IGWs are not directly excited by the forcing, they are generated via nonlinear interactions with the slower geostrophic motion. Indeed, their generation and amplitude seem to influence the transition to a mesoscale $-5 / 3$ spectrum.

Is white noise the best approach for forcing geostrophic motion in rotating stratified turbulence? Forcing all time scales is not a particularly realistic model for the injection of energy from larger scale balanced motion. The forced geostrophic modes evolve on the slow advective time scale, but white 
noise forcing excites high frequencies as well. It is possible that, since the geostrophic modes evolve slowly, any high frequencies in the forcing might be benign and not contribute much to the energy injection rate. On the other hand, the geostrophic modes are coupled to wave modes via the nonlinearities of the equations of motion. As a result, high frequencies in the forcing may be communicated to the fast modes, thereby influencing the injection of energy into the waves. This possibility is investigated here, by employing red noise forcing with different decorrelation times. The background and methodology are outlined in Sec. II, results are presented in Sec. III, and conclusions are given in Sec. IV.

\section{APPROACH}

\section{A. Equations}

For this study, we use the Boussinesq equations on an $f$-plane with constant buoyancy frequency $N$,

$$
\begin{aligned}
\frac{\mathrm{D} \boldsymbol{u}}{\mathrm{D} t}+f \boldsymbol{z} \times \boldsymbol{u} & =-\nabla p+b \hat{z}+\boldsymbol{F}_{u}+\boldsymbol{D}_{u}, \\
\frac{\mathrm{D} b}{\mathrm{D} t}+N^{2} w & =F_{b}+D_{b}, \\
\nabla \cdot \boldsymbol{u} & =0,
\end{aligned}
$$

where $\boldsymbol{u}$ is the velocity, $p$ is the pressure scaled by a reference density, and $b$ is the buoyancy. The velocity and buoyancy forcing $\boldsymbol{F}_{u}$ and $F_{b}$ and diffusion $\boldsymbol{D}_{u}$ and $D_{b}$ are given below. Boundary conditions are periodic in the horizontal and vertical directions. Since the buoyancy $b$ is proportional to the fluctuation potential temperature about a linearly varying basic state, it is only the fluctuation potential temperature, not the total, that is assumed to be periodic.

With periodic boundary conditions, Eqs. (1)-(3) can be Fourier transformed into equations for the Fourier coefficients $\hat{u}_{i}(\boldsymbol{k}, t)$ and $\hat{b}(\boldsymbol{k}, t)$, where $\boldsymbol{k}=\left(k_{x}, k_{y}, k_{z}\right)$ is the discrete three-dimensional wave vector. The dependence on time $t$ is suppressed for clarity. These equations can be rewritten in terms of the linear normal mode basis at every wave vector,, $18-20$

$$
\begin{aligned}
\frac{\partial}{\partial t} B_{\boldsymbol{k}}^{(j)}+\mathrm{i} \lambda_{\boldsymbol{k}}^{(j)} B_{\boldsymbol{k}}^{(j)}= & \sum_{\boldsymbol{p}, \boldsymbol{q}, \boldsymbol{r}, s} \Gamma_{\boldsymbol{k} p \boldsymbol{q}}^{j r s} B_{\boldsymbol{p}}^{(r)} B_{\boldsymbol{q}}^{(s)} \\
& +F_{\boldsymbol{k}}^{(j)}+D_{\boldsymbol{k}}^{(j)}
\end{aligned}
$$

where $j, r, s \in\{-1,0,1\}$ and the sum on the RHS is over wave vector triads $\boldsymbol{k}=\boldsymbol{p}+\boldsymbol{q}$. Here, $B_{\boldsymbol{k}}^{(j)}$ is the amplitude of normal mode $j$ at wave vector $\boldsymbol{k}$, where $j=0$ corresponds to the geostrophic mode and $j= \pm 1$ corresponds to the IGW mode with positive and negative frequency, respectively. The $\Gamma_{k p q}^{j r s}$ are the interaction coefficients. The linear frequencies $\lambda_{k}^{(j)}$ are given by the dispersion relation

$$
\lambda_{\boldsymbol{k}}^{(0)}=0, \quad \lambda_{\boldsymbol{k}}^{( \pm 1)}= \pm \frac{\sqrt{N^{2} k_{h}^{2}+f^{2} k_{v}^{2}}}{k},
$$

where $k_{h}^{2}=k_{x}^{2}+k_{y}^{2}, k_{v}=\left|k_{z}\right|$, and $k^{2}=k_{h}^{2}+k_{v}^{2}$. The velocity and buoyancy for the geostrophic mode are in geostrophic and thermal wind balance; the wave modes contain all the horizontal divergence and have velocities and buoyancy in the correct phase relationships for linear plane waves with no background flow. The evolution of mode $B_{\boldsymbol{k}}^{(j)}$ is a linear oscillation at a natural frequency (zero for the geostrophic mode and given by the IGW dispersion relation for the wave modes) along with triad interactions with other modes. The normal mode decomposition is convenient because of its simplicity and orthogonality; e.g., it allows for a clean decomposition of the total energy into geostrophic and wave contributions. ${ }^{9}$ However, as a linear decomposition, it does not isolate balanced motion as well as higher-order balance schemes..$^{21}$ The projection of the forcing and dissipation onto the modes are given by $F_{\boldsymbol{k}}^{(j)}$ and $D_{\boldsymbol{k}}^{(j)}$, respectively.

Because of the orthogonality of the modes, the total mechanical energy $E(\boldsymbol{k})$ in the wave vector $\boldsymbol{k}$ satisfies

$$
\begin{aligned}
2 E(\boldsymbol{k}) & =\underbrace{|\hat{u}(\boldsymbol{k})|^{2}+|\hat{v}(\boldsymbol{k})|^{2}+|\hat{w}(\boldsymbol{k})|^{2}}_{\text {kinetic }}+\underbrace{\frac{|\hat{b}(\boldsymbol{k})|^{2}}{N^{2}}}_{\text {potential }} \\
& =\underbrace{\left|B^{(0)}(\boldsymbol{k})\right|^{2}}_{\text {geostrophic }}+\underbrace{\left|B^{(-1)}(\boldsymbol{k})\right|^{2}+\left|B^{(+1)}(\boldsymbol{k})\right|^{2}}_{\text {wave }}
\end{aligned}
$$

and therefore can be decomposed into a sum of kinetic and potential energy, as well as a sum of geostrophic and IGW energy. ${ }^{9}$ The evolution of the geostrophic and wave modal energies at wave vector $\boldsymbol{k}$ is obtained by multiplying (4) by $B_{k}^{(j) *}$, where * denotes the complex conjugate, adding the complex conjugate equation, and summing the +1 and -1 wave energies together. The resulting equations are

$$
\begin{gathered}
\frac{\partial}{\partial t} \frac{1}{2}\left|B_{\boldsymbol{k}}^{(0)}\right|^{2}=T_{\boldsymbol{k}}^{(0)}+\mathcal{F}_{\boldsymbol{k}}^{(0)}+\mathcal{D}_{\boldsymbol{k}}^{(0)}, \\
\frac{\partial}{\partial t} \frac{1}{2}\left(\left|B_{\boldsymbol{k}}^{(+)}\right|^{2}+\left|B_{\boldsymbol{k}}^{(-)}\right|^{2}\right) \\
=T_{\boldsymbol{k}}^{(W)}+\mathcal{F}_{\boldsymbol{k}}^{(W)}+\mathcal{D}_{\boldsymbol{k}}^{(W)},
\end{gathered}
$$

where $T, \mathcal{F}$, and $\mathcal{D}$ terms are nonlinear transfer, forcing, and dissipation, respectively. Here $W$ denotes wave, i.e., the sum of the +1 and -1 contributions. The nonlinear transfer terms are sums over normal mode triads and therefore have contributions from the various classes of geostrophic-geostrophic, geostrophic-wave, and wave-wave interactions. ${ }^{9}$ In particular, the transfer term in the wave equation is

$$
\begin{aligned}
T_{\boldsymbol{k}}^{(W)} & =\sum_{\boldsymbol{p}, \boldsymbol{q}, r, s}\left(\Gamma_{\boldsymbol{k} \boldsymbol{p} \boldsymbol{q}}^{+1 r s} B_{\boldsymbol{k}}^{(+1) *}+\Gamma_{\boldsymbol{k} \boldsymbol{p} \boldsymbol{q}}^{-1 r s} B_{\boldsymbol{k}}^{(-1) *}\right) B_{\boldsymbol{p}}^{(r)} B_{\boldsymbol{q}}^{(s)}+c . c . \\
& =T^{(W G G)}+T^{(W W G)}+T^{(W W W)} .
\end{aligned}
$$

The wave energy transfer has contributions from wavegeostrophic-geostrophic (WGG) interactions $(r=s=0)$, wave-wave-geostrophic (WWG) interactions (one of $r$ and $s=0$, the other $= \pm 1$ ), and wave-wave-wave (WWW) interactions (both $r$ and $s= \pm 1$ ). The geostrophic energy transfer has a similar decomposition. In strongly rotating and stratified flows, the WWG and WWW terms are dominated by resonant triads, ${ }^{9}$ i.e., triads for which $\lambda_{k}^{(j)}+\lambda_{p}^{(r)}+\lambda_{q}^{(s)}=0$. In resonant WWG interactions, the geostrophic mode facilitates a transfer of energy between the two wave modes in the triad but is unaffected itself. ${ }^{9,19,22}$ The transfer of energy 
between geostrophic and wave modes is therefore mainly given by the WGG term, which are never resonant because the geostrophic frequencies are both zero. Off-resonant WWG interactions may also contribute to geostrophic-wave exchange. ${ }^{9}$

\section{B. Numerical model}

Equations (1)-(3) are solved with a Fourier-based spectral code on a domain of size $L \times L \times H$, where $L=4000 \mathrm{~km}$ and $H=20 \mathrm{~km}$. Time stepping is performed in the spectral domain with the third-order Adams-Bashforth scheme with constant time step $\Delta t$, and dissipation terms are treated implicitly with a Crank-Nicolson approach. ${ }^{23}$ Nonlinear terms are computed in physical space and transformed back and forth with a fast Fourier transform. ${ }^{24}$ The number of wavenumbers is $n_{h} \times n_{h} \times n_{v}$ and aliasing errors are eliminated by axisymmetric truncation following the two-thirds rule. ${ }^{25}$ As a result, the effective grid spacings are

$$
\Delta x=\Delta y \approx 1.5 L / n_{h}, \quad \Delta z \approx 1.5 H / n_{v} .
$$

The wavenumber spacing is $\Delta k_{h}=2 \pi / L$ in the horizontal and $\Delta k_{v}=2 \pi / H$ in the vertical. Dimensionless (integer) wavenumbers $k_{h}^{\prime}=k_{h} / \Delta k_{h}$ and $k_{z}^{\prime}=k_{z} / \Delta k_{v}$ are employed below for convenience. Spectral quantities are computed in terms of $k_{h}$ by summing wave vectors in $k_{h}$ bins of width $\Delta k_{h}$, i.e., for geostrophic energy

$$
E^{(G)}\left(k_{h}^{\prime}\right) \Delta k_{h}=\sum_{k_{h}^{\prime}-1 / 2 \leq p_{h}^{\prime} \leq k_{h}^{\prime}+1 / 2} \frac{1}{2}\left|B^{(0)}(\boldsymbol{p})\right|^{2},
$$

for integer values of $k_{h}^{\prime}$ and similarly for the IGW energy spectrum $E^{(W)}\left(k_{h}^{\prime}\right)$.

At geophysical scales, direct numerical simulation is not feasible. As an alternative, we use hyperviscosity and hyperdiffusion to dissipate energy at small scales, which is a common approach in idealized studies of rotating stratified turbulence..$^{3,10,11,13,14}$ The dissipation operators in (1)-(3) are

$$
\boldsymbol{D}_{u}=-v \nabla_{h}^{8} \boldsymbol{u}, \quad D_{b}=-\kappa \nabla_{h}^{8} b .
$$

No explicit vertical dissipation is included, which is reasonable when the vertical resolution is not too low. ${ }^{7}$ Hyperdiffusion coefficients are set by assuming hyper-Prandtl number of unity, i.e., $\kappa=v$. In addition to the hyperviscosity, there is also a weak linear damping term, with time scale 1 day, applied to modes with $k_{h}^{\prime} \leq 3$ to balance any inverse cascade.

\section{Forcing}

The forcing is designed to excite large-scale geostrophic modes, with

$$
F_{\boldsymbol{k}}^{(0)}=\left\{\begin{array}{ll}
A G\left(\boldsymbol{k}, t_{n}\right) & k_{h}^{\prime} \in[3,5], \quad k_{v}^{\prime}=1 \\
0 & \text { otherwise }
\end{array}, \quad F_{\boldsymbol{k}}^{( \pm 1)}=0,\right.
$$

at time $t_{n}=n \Delta t$, where $A \in \mathbb{R}$ is the amplitude. There are $n_{f}=108$ wave vectors in the forcing band $k_{h}^{\prime} \in[3,5], k_{v}^{\prime}=1$. The time dependence is chosen to be an AR(1) process with specified decorrelation time scale $\tau,{ }^{15,26,27}$

$$
G\left(\boldsymbol{k}, t_{n}\right)=\alpha G\left(\boldsymbol{k}, t_{n-1}\right)+\beta g(\boldsymbol{k}, n),
$$

where $g(\boldsymbol{k}, n)$ is a random complex number having Gaussian real and imaginary parts with zero mean and unit variance. The same random sequence $g(\boldsymbol{k}, n)$ is used in each simulation. The coefficients satisfy $\alpha=\exp (-\Delta t / \tau)$ and $\beta^{2}=1-\alpha^{2}$, implying that $G\left(\boldsymbol{k}, t_{n}\right)$ has variance 2 (sum of the real and imaginary variances). The time scale $\tau$ sets all the parameters in (14). The interpretation of $\tau$ can be seen by considering the autocovariance

$$
\begin{aligned}
\left\langle G\left(\boldsymbol{k}, t_{n+m}\right) G\left(\boldsymbol{k}, t_{n}\right)\right\rangle & =2 \alpha^{m} \\
& =2 \exp (-m \Delta t / \tau),
\end{aligned}
$$

where $\langle\ldots\rangle$ denotes the ensemble average (time averages are used below). The autocovariance falls off exponentially with time scale $\tau$. Letting $\tau \rightarrow 0$ implies $\alpha \rightarrow 0$ and the forcing is white noise. On the other hand, setting $\tau \gg \Delta t$ gives $\alpha \gg \beta$ and the forcing is correlated over many time steps. We use $\tau \geq 3 \Delta t$ so that the forcing is resolved by the time stepping scheme.

The average rate of the energy input by the forcing is $\langle P\rangle$, where

$$
P=\frac{1}{2} \sum_{\boldsymbol{k}} F_{\boldsymbol{k}}^{(0)} B_{\boldsymbol{k}}^{(0) *}+c . c .
$$

The forcing amplitude is chosen to give approximately equal $\langle P\rangle$ for all $\tau$. Let $A=a / \sqrt{\tau}$. For small $\tau$, equal $a$ values yield approximately equal $\langle P\rangle \approx 2 n_{f} a^{2}$, similar to the white noise case. However, for larger $\tau$, the $1 / \sqrt{\tau}$ dependence causes $\langle P\rangle$ to decrease with increasing $\tau$ when $a$ is held fixed. As a result, for larger $\tau$, we increase $a$ to keep $\langle P\rangle$ within $2 \%$ of the small $\tau$ value $P_{f}$.

\section{Setup of simulations}

Our main set of simulations is run for a range of $\tau$ with $n_{h}=256, n_{v}=128, f=10^{-4} \mathrm{~s}^{-1}, N=10^{-2} \mathrm{~s}^{-1}, \Delta t=15 \mathrm{~s}$, and $v=4.8 \times 10^{28} \mathrm{~m}^{8} \mathrm{~s}^{-1}$. The grid resolution is therefore $\Delta x=23.8 \mathrm{~km}$ and $\Delta z=238 \mathrm{~m}$. The corresponding inertial period is $17 \mathrm{~h}$. The target forcing power $P_{f}=8.4 \times$ $10^{-4} \mathrm{~m}^{2} \mathrm{~s}^{-3}$. Forcing parameters for each $\tau$ are given in Table I. Parameters are chosen to be typical of the atmospheric mesoscale at mid-latitudes. The simulations are characterized by the forcing length scales $L_{f}=L / 4=1000 \mathrm{~km}$ and $H_{f}=H=20 \mathrm{~km}$ and velocity scale $U_{f}=\left(L_{f} P_{f}\right)^{1 / 3}=9.4 \mathrm{~ms}^{-1}$. The corresponding time scale of the forced vortices is $T_{f}=$ $L_{f} / U_{f}=29 \mathrm{~h}$. The associated Rossby and Froude numbers are

$$
R o=\frac{U_{f}}{f L_{f}}=0.094, \quad F r=\frac{U_{f}}{N H_{f}}=0.047 .
$$

A range of $\tau$ values between $\Delta t$ and $2 T_{f}$ is considered. Simulations are initialized with low-level random large-scale geostrophic modes and run for 48 days $=40 T_{f}$, except for simulations with $\tau=48 \mathrm{~h}$ and $72 \mathrm{~h}$, which are run for 72 days $=60 T_{f}$. Statistics are averaged over the entire simulation after a 6 day spin-up period.

In addition, two sets of sensitivity simulations are performed. In the first set, the dependence on resolution is tested by repeating the $\tau=150 \mathrm{~s}$ and $24 \mathrm{~h}$ simulations with $n_{h}=512$. The fast forcing simulation with $\tau=150 \mathrm{~s}$ is also performed 
TABLE I. Summary of forcing amplitude $a$ and power $\langle P\rangle$ for simulations with $n_{h}=256, n_{v}=128, f=10^{-4} \mathrm{~s}^{-1}$, and $N=10^{-2} \mathrm{~s}^{-1}$.

\begin{tabular}{lcccc}
\hline \hline$\tau$ & $\tau f$ & $a\left(\mathrm{~ms}^{-3 / 2}\right)$ & $\langle P\rangle\left(\mathrm{m}^{2} \mathrm{~s}^{-3}\right)$ & $\langle P\rangle /\left(2 n_{f} a^{2}\right)$ \\
\hline $45 \mathrm{~s}$ & 0.0045 & $1.92 \times 10^{-3}$ & $8.34 \times 10^{-4}$ & 1.0 \\
$150 \mathrm{~s}$ & 0.0150 & $1.92 \times 10^{-3}$ & $8.36 \times 10^{-4}$ & 1.1 \\
$5 \mathrm{~min}$ & 0.0300 & $1.92 \times 10^{-3}$ & $8.36 \times 10^{-4}$ & 1.1 \\
$10 \mathrm{~min}$ & 0.0600 & $1.92 \times 10^{-3}$ & $8.20 \times 10^{-4}$ & 1.0 \\
$15 \mathrm{~min}$ & 0.0900 & $1.92 \times 10^{-3}$ & $8.41 \times 10^{-4}$ & 1.1 \\
$30 \mathrm{~min}$ & 0.180 & $1.92 \times 10^{-3}$ & $8.40 \times 10^{-4}$ & 1.1 \\
$1 \mathrm{~h}$ & 0.360 & $1.92 \times 10^{-3}$ & $8.23 \times 10^{-4}$ & 1.0 \\
$3 \mathrm{~h}$ & 1.08 & $2.00 \times 10^{-3}$ & $8.18 \times 10^{-4}$ & 0.95 \\
$6 \mathrm{~h}$ & 2.16 & $2.15 \times 10^{-3}$ & $8.39 \times 10^{-4}$ & 0.84 \\
$12 \mathrm{~h}$ & 4.32 & $2.38 \times 10^{-3}$ & $8.48 \times 10^{-4}$ & 0.69 \\
$24 \mathrm{~h}$ & 8.64 & $2.76 \times 10^{-3}$ & $8.29 \times 10^{-4}$ & 0.50 \\
$48 \mathrm{~h}$ & 17.3 & $3.32 \times 10^{-3}$ & $8.16 \times 10^{-4}$ & 0.34 \\
$72 \mathrm{~h}$ & 25.9 & $3.88 \times 10^{-3}$ & $8.43 \times 10^{-4}$ & 0.26 \\
\hline \hline
\end{tabular}

TABLE II. Summary of parameters used in sensitivity tests with $n_{h}=256$, $n_{v}=128, f=2 \times 10^{-4} \mathrm{~s}^{-1}$, and $N=2 \times 10^{-2} \mathrm{~s}^{-1}$.

\begin{tabular}{lcccc}
\hline \hline$\tau$ & $\tau f$ & $a\left(\mathrm{~ms}^{-3 / 2}\right)$ & $\langle P\rangle\left(\mathrm{m}^{2} \mathrm{~s}^{-3}\right)$ & $\langle P\rangle /\left(2 n_{f} a^{2}\right)$ \\
\hline $45 \mathrm{~s}$ & 0.00900 & $1.92 \times 10^{-3}$ & $8.43 \times 10^{-4}$ & 1.1 \\
$150 \mathrm{~s}$ & 0.0300 & $1.92 \times 10^{-3}$ & $8.54 \times 10^{-4}$ & 1.1 \\
$5 \mathrm{~min}$ & 0.0600 & $1.92 \times 10^{-3}$ & $8.13 \times 10^{-4}$ & 1.0 \\
$15 \mathrm{~min}$ & 0.180 & $1.92 \times 10^{-3}$ & $8.38 \times 10^{-4}$ & 1.1 \\
$30 \mathrm{~min}$ & 0.360 & $1.92 \times 10^{-3}$ & $8.11 \times 10^{-4}$ & 1.0 \\
$1 \mathrm{~h}$ & 0.720 & $1.92 \times 10^{-3}$ & $8.15 \times 10^{-4}$ & 1.0 \\
$3 \mathrm{~h}$ & 2.16 & $2.00 \times 10^{-3}$ & $8.34 \times 10^{-4}$ & 0.96 \\
$6 \mathrm{~h}$ & 4.32 & $2.15 \times 10^{-3}$ & $8.40 \times 10^{-4}$ & 0.84 \\
$12 \mathrm{~h}$ & 8.64 & $2.38 \times 10^{-3}$ & $8.71 \times 10^{-4}$ & 0.71 \\
$24 \mathrm{~h}$ & 17.3 & $2.76 \times 10^{-3}$ & $8.42 \times 10^{-4}$ & 0.51 \\
\hline \hline
\end{tabular}

with $n_{h}=1024$. Higher-resolution simulations are only run for $24 \mathrm{~h}$. The hyperviscosity coefficient is adjusted to keep the grid scale damping rates fixed $(\approx 200 \mathrm{~s})$. In the second set, the dependence on $f$ and $N$ is investigated by repeating simulations with $f=2 \times 10^{-4} \mathrm{~s}^{-1}$ and $N=2 \times 10^{-2} \mathrm{~s}^{-1}$ with $n_{h}=256$ for a subset of $\tau$ (Table II). In all sensitivity tests, the forcing amplitudes from Table I are used. The resulting $P$ values are within a few percent of the values in Table I.

\section{RESULTS}

\section{A. Dependence on $\tau$}

Time series of domain-averaged total energy $E=E^{(G)}+E^{(W)}$ are shown in Fig. 1 for a subset of the $\tau$ values. Here $E^{(G)}$ and $E^{(W)}$ are the sum over $\boldsymbol{k}$ of the modal energies $\left|B_{\boldsymbol{k}}^{(0)}\right|^{2}$ and $\left|B_{\boldsymbol{k}}^{( \pm 1)}\right|^{2}$, respectively. Simulations are started from near rest, and it takes a few days for the energy to approach its statistical equilibrium value. The time series appear stationary for $t \gtrsim 6$ days, and all $\tau$ values give approximately equal timeaveraged energy $\langle E\rangle \approx 110 \mathrm{~m}^{2} \mathrm{~s}^{-2}$. The average kinetic energy is similarly insensitive to $\tau$, and the square root of the average $\mathrm{KE}$ is around $9.5 \mathrm{~ms}^{-1}$, which is close to the forcing velocity

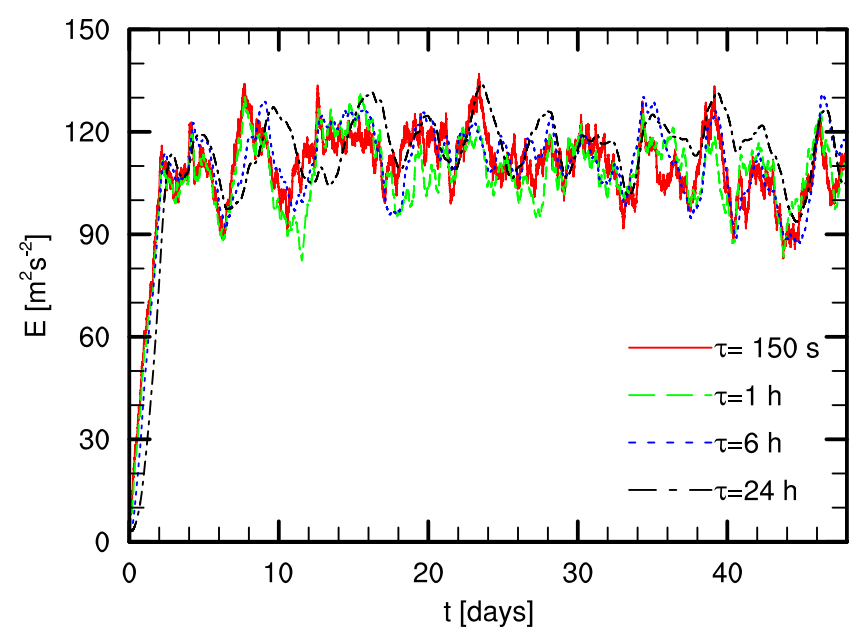

FIG. 1. Time series of total energy (i.e., kinetic plus potential or equivalently geostrophic plus wave) in simulations with $\tau=150 \mathrm{~s}, 1 \mathrm{~h}, 6 \mathrm{~h}$, and $24 \mathrm{~h}$.

$U_{f}$ found above. However, the signature of the forcing time scale is clear in the time series, where the large- $\tau$ simulations vary much more slowly than those with small $\tau$.

Time series of instantaneous forcing power $P$ scaled by $P_{f}$ are shown in Fig. 2. For $\tau \leq 1 \mathrm{~h}$, only a $6 \mathrm{~h}$ segment is shown
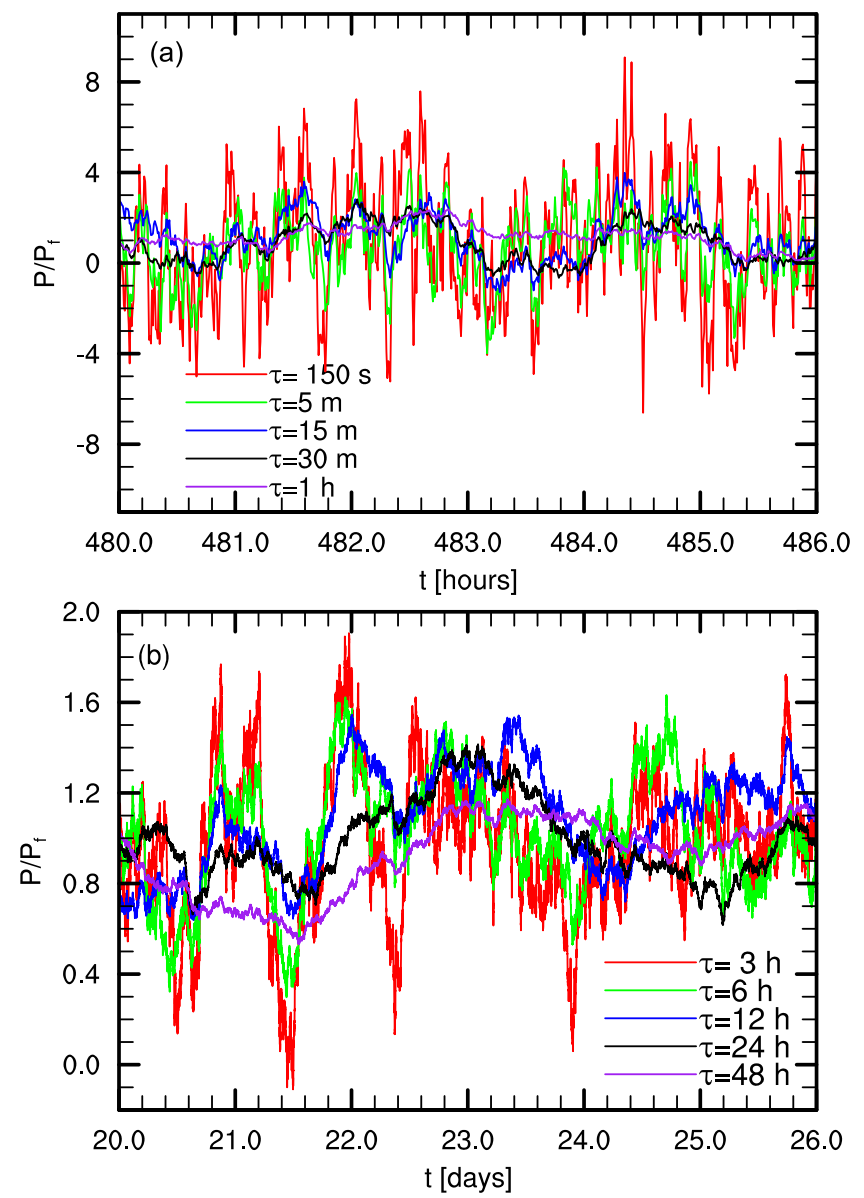

FIG. 2. Time series of forcing power in simulations with (a) $\tau=150 \mathrm{~s}, 5 \mathrm{~m}$, $15 \mathrm{~m}, 30 \mathrm{~m}$, and $1 \mathrm{~h}$, and (b) $3 \mathrm{~h}, 6 \mathrm{~h}, 12 \mathrm{~h}, 24 \mathrm{~h}$, and $48 \mathrm{~h}$. Note that the $t$-axes in (a) and (b) are different to better illustrate the high frequencies in the time series. 
for clarity; for longer $\tau, 6$ days are presented. As for the total energy, the time scale of the forcing is clearly visible in these time series, with $P$ evolving much more rapidly for small $\tau$ than for large. In addition, the variance of $P$ is much larger for small $\tau$, especially for $\tau=45 \mathrm{~s}$ and $150 \mathrm{~s}$. The variance decreases with increasing $\tau$. In particular, the variance is $\approx 5 P_{f}$ for $\tau=45 \mathrm{~s}$ and only $0.2 P_{f}$ for $\tau=24$ hours. As $\tau$ increases, the forcing becomes better correlated with the flow, and the instantaneous power remains closer to its average value. Recall that in all
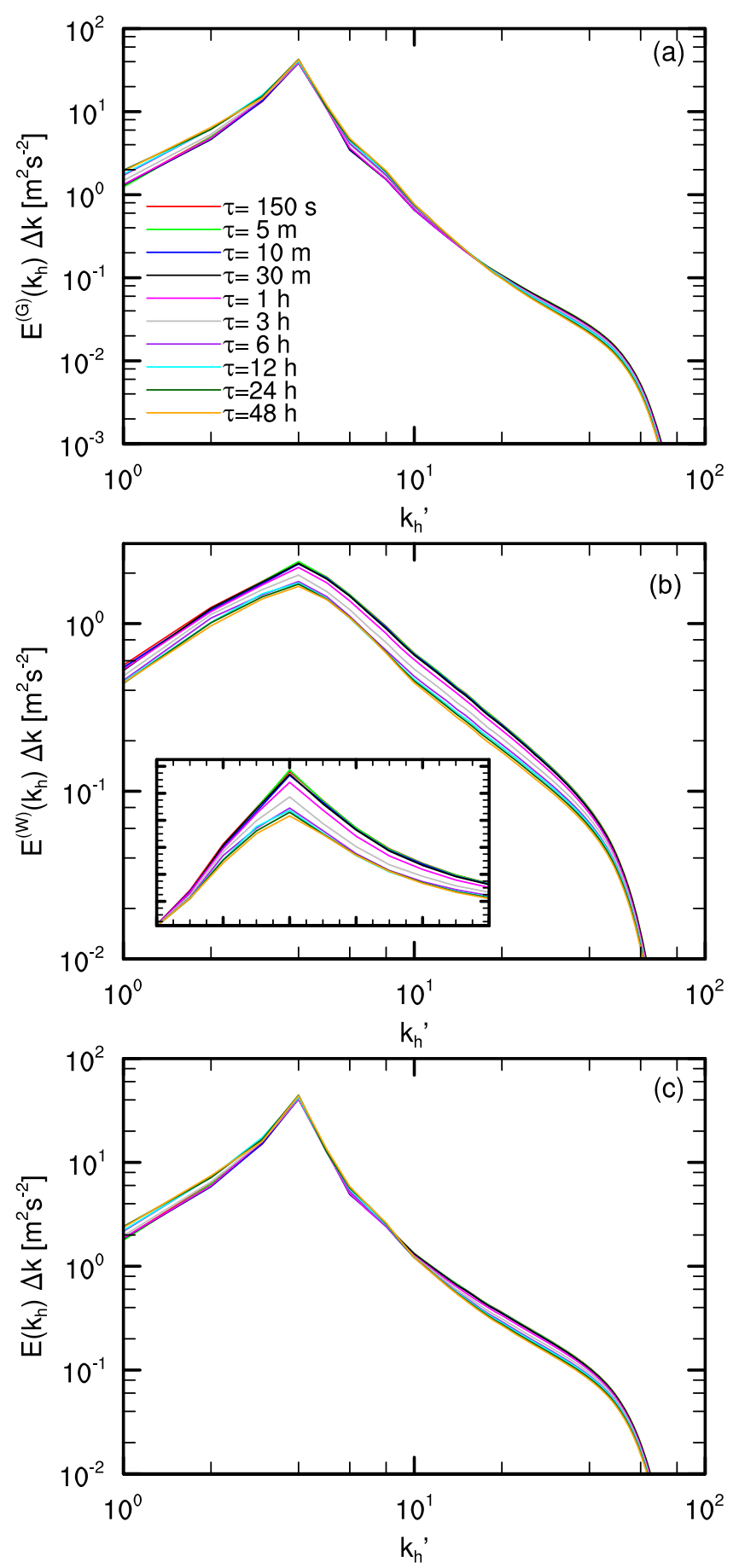

FIG. 3. Time-averaged horizontal wavenumber spectra of (a) geostrophic, (b) wave, and (c) total energy for all $\tau$. In (b), the inset shows the wave spectra with linear-linear axes for $0 \leq k_{h} \leq 20$. cases, the time-averaged power is approximately $P_{f}$, so the average of all the time series in Fig. 2 is close to 1.

Time-averaged $k_{h}$ spectra of geostrophic, wave, and total energy are shown in Fig. 3. The geostrophic spectra [Fig. 3(a)] are nearly identical for all $\tau$, suggesting that the geostrophic energy spectrum is nearly independent of the forcing time scale. All spectra exhibit a peak in the forcing wavenumber band $3 \leq k_{h} \leq 5$, followed by a power law range with a slope around -3.2 , followed by a narrow dissipation range where the energy falls off rapidly with increasing $k_{h}$. There is some shallowing in the geostrophic spectra at larger $k_{h}$ before the dissipation range. By contrast, the amplitudes of the wave spectra [Fig. 3(b)] vary with $\tau$, with smaller $\tau$ having larger amplitudes. In all cases, the wave spectra are peaked around the forcing band and fall off with a power law range with a slope of around -1.5 . The geostrophic and wave spectral slopes are therefore approximately -3 and $-5 / 3$, in agreement with previous work. ${ }^{15}$ However, Fig. 3(b) indicates that the time scale of the forcing does influence the amount of wave energy that is generated, with shorter $\tau$ simulations being more effective at generating waves. The effect on the spectrum of total energy [Fig. 3(c)] is small.

To further explore the dependence of the wave energy on $\tau$, Fig. 4 plots the time-averaged fraction of wave energy $E^{(W)} / E$ for different $\tau$. The $n_{h}=256$ simulations are plotted in red. For $\tau \lesssim 1800 \mathrm{~s}=30 \mathrm{~min}$, the wave energy is approximately constant at $18.7 \%$ of the total. As $\tau$ increases further, this fraction decreases to as low as $12.8 \%$ for $\tau=48$ and $72 \mathrm{~h}$. In other words, the small- $\tau$ simulations have around $46 \%$ more wave energy than the largest $\tau$ case. The fraction of wave energy seems to be levelling off for large $\tau$. The large- $\tau$ ratio is consistent with numerical studies from more comprehensive global atmospheric models, which show a ratio of divergent to total kinetic energy at $200 \mathrm{hPa}$ of around $10 \%$ at length scales of $1000 \mathrm{~km} .{ }^{28}$ Note, however, that these ratios are not exactly equivalent because the geostrophic and wave modes decompose the total (kinetic and potential) energy, while the rotational and divergent energy decompose only the kinetic energy.

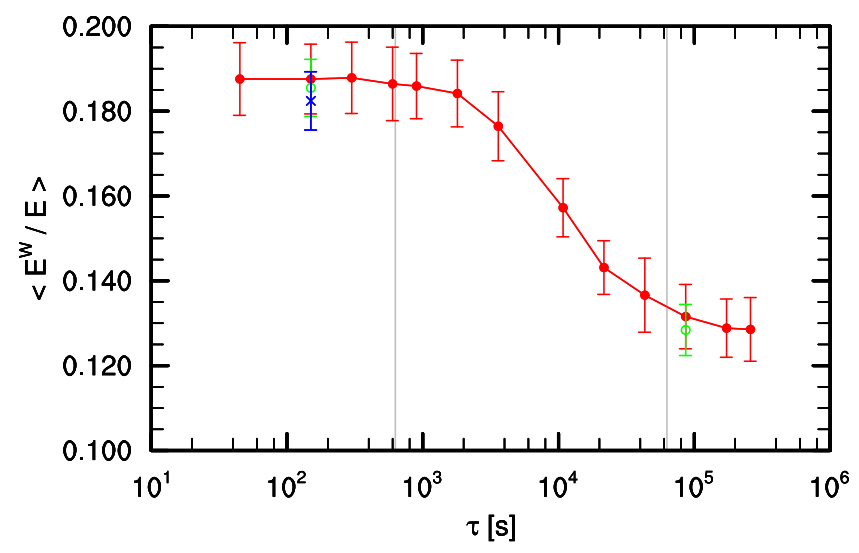

FIG. 4. Time-averaged ratio of wave energy to total energy for simulations with different $\tau$ and different grid resolutions. Colors correspond to $n_{h}=256$ (red closed circles), 512 (green open circles), and 1024 (blue x). Error bars show \pm 1 standard deviation in the time series. The vertical lines mark the buoyancy and inertial periods. 
Wave energy is not directly forced but is rather generated via nonlinear interactions with the geostrophic motion. To quantify this process, we consider the terms in the wave energy transfer (9). Figure 5 shows the contribution to the wave energy transfer from wave-geostrophic-geostrophic, wave-wave-geostrophic, and wave-wave-wave interactions. Total integrated WGG and WWG transfer, which give the net conversion rate of geostrophic to wave energy, are given in Fig. 6 (the WWW interactions are not shown because they are conservative and sum to zero). The production of wave energy from geostrophic modes is dominated by the WGG
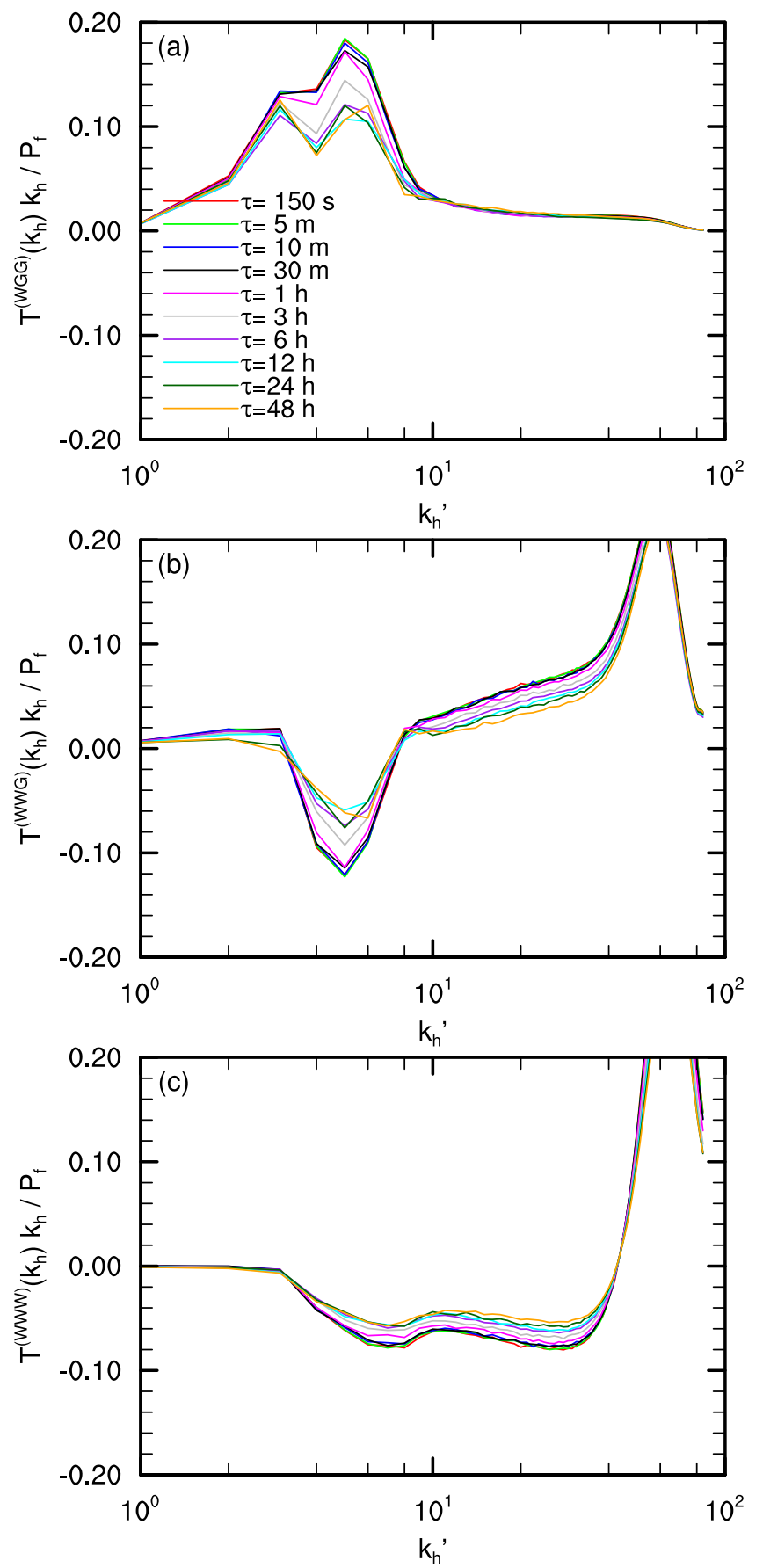

FIG. 5. Time-averaged $k_{h}$ wave energy transfer spectra due to (a) WGG, (b) WWG, and (c) WWW interactions for all $\tau$. Spectra are multiplied by $k_{h}$ to preserve area on log-linear axes and are nondimensionalized by $P_{f}$.

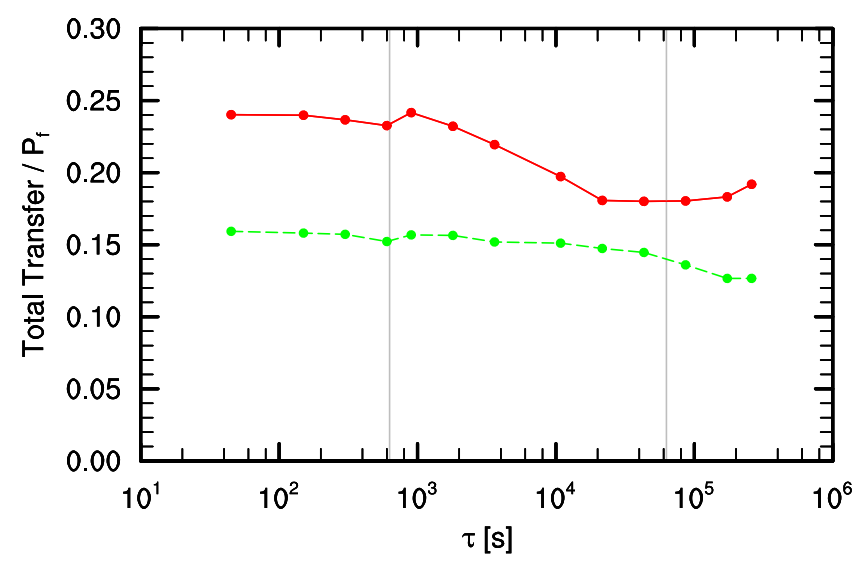

FIG. 6. Total transfer due to wave-geostrophic-geostrophic (red, solid) and wave-wave-geostrophic (green, dashed) interactions, i.e., the sum of the spectra in Figs. 5(a) and 5(b) over $k_{h}$. The vertical lines mark the buoyancy and inertial periods.

interactions [Fig. 5(a)]. This term is everywhere positive and peaked in the forcing band. There is a clear dependence on $\tau$, with the smallest $\tau$ values having the largest transfer values, and a general decreasing trend as $\tau$ increases. This trend is evident in Fig. 6, where the total WGG transfer is 25\%-30\% larger for the smallest $\tau$ than for $\tau \gtrsim 6 \mathrm{~h}$. There is also a smaller net conversion of geostrophic to wave energy from the WWG interactions, which decreases only slightly with increasing $\tau$ (Fig. 6). Therefore, despite the random forcing of geostrophic modes having the same power for all $\tau$, the transfer into the wave energy is more efficient for smaller $\tau$. Once injected, the wave energy is removed from the forcing band, cascades downscale, and is deposited in the dissipation range by the WWG and WWW interactions.

To better understand the role that $\tau$ plays in geostrophicwave interactions, we consider the frequency spectra of a subset of modal amplitudes $B_{\boldsymbol{k}}^{(j)}$. We consider nine $\boldsymbol{k}$ from the forcing band and present average frequency spectra of the associated time series over the averaging interval $6 \leq t \leq 48$ days. A Hanning window was used to make the time series periodic. Frequencies are nondimensionalized by the interval length, i.e., $\omega^{\prime}=\omega / \Delta \omega$, where $\Delta \omega=2 \pi /(42$ days $)$. Frequency spectra are shown in Figs. 7(a) and 7(b) where, as above, the +1 and -1 spectra are summed to get the wave spectra. In addition, wave spectra for nine $k$ with $k_{h}$ in the forcing band and $k_{v}^{\prime}=2$ are also shown [Fig. 7(c)], since these are wave modes that will interact with forced geostrophic modes. The linear wave frequencies $\omega_{L I N} \approx 130=2.2 f$ and $80=1.4 f$ associated with $k_{v}^{\prime}=1$ and 2 are indicated in Figs. 7(b) and 7(c). The corresponding wave periods are $8 \mathrm{~h}$ for $k_{v}^{\prime}=1$ and $12 \mathrm{~h}$ for $k_{v}^{\prime}=2$.

The geostrophic frequency spectra [Fig. 7(a)] are fairly flat and independent of $\tau$ for the lowest frequencies $\omega^{\prime} \lesssim 10$, corresponding to periods $\gtrsim 4$ days. For higher frequencies, there is a significant dependence on $\tau$, with the spectra for smaller $\tau$ being much broader and shallower than those for longer $\tau$. The forced geostrophic modes have significantly more energy at high frequencies when the forcing is faster, as expected. This dependence is dramatic: for example, around $\omega^{\prime}=1000$ (period of $1 \mathrm{~h}$ ), the geostrophic energy is roughly constant for 

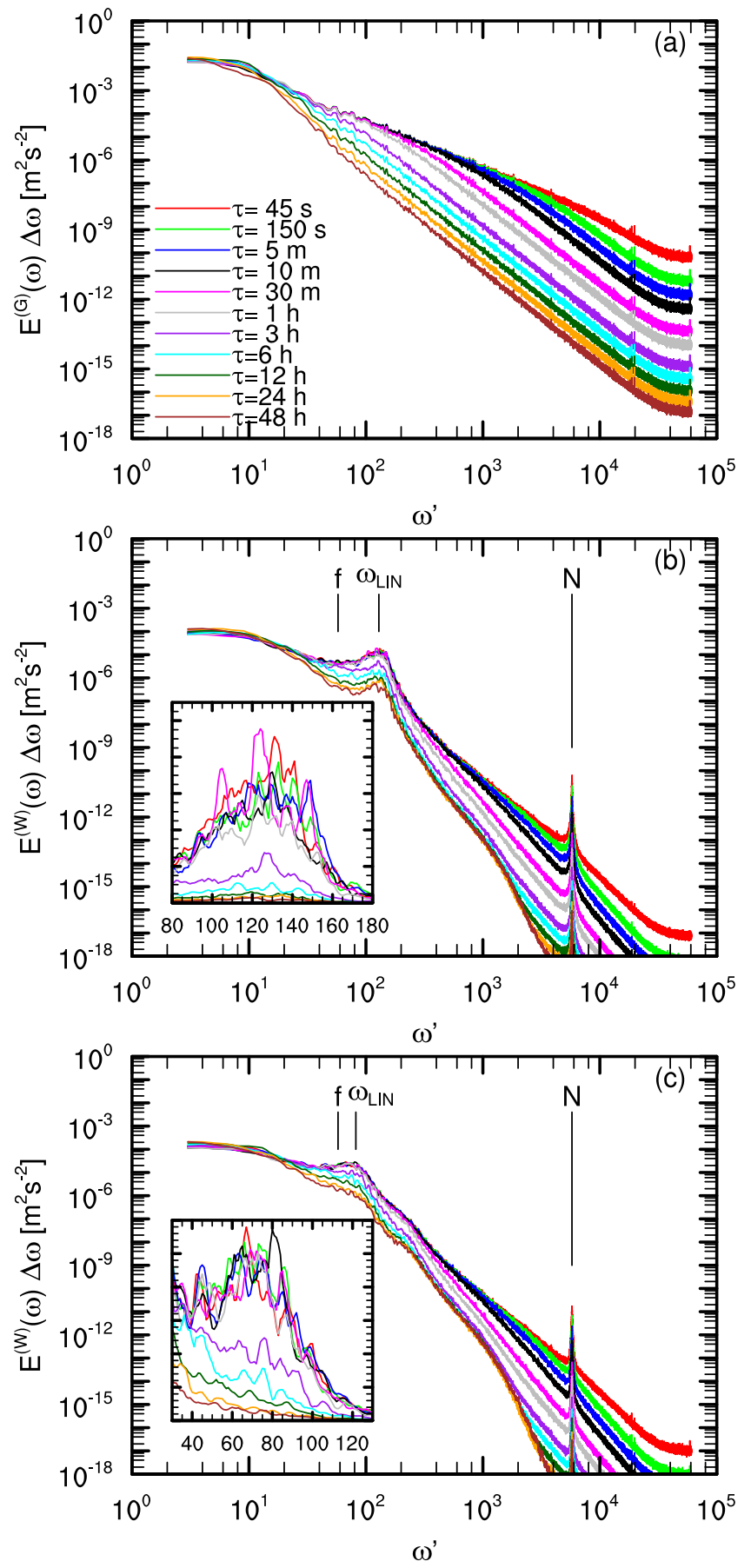

FIG. 7. Average frequency spectra of (a) geostrophic and (b) wave modes for nine wave vectors with $k_{h}^{\prime} \approx 4$ and $k_{v}^{\prime}=1$, and (c) wave modes for nine wave vectors with $k_{h} \approx 4$ and $k_{v}^{\prime}=2$. Dimensionless frequency $\omega^{\prime}$ corresponds to a period of $42 / \omega^{\prime}$ days. Spectra are smoothed with a 5-point running average. The linear wave frequency $\omega_{\text {LIN }}$, buoyancy frequency $N$, and Coriolis frequency $f$ are marked for reference. In (b) and (c), the inset shows the region around $\omega=\omega_{L I N}$ with linear-linear axes.

$\tau=45 \mathrm{~s}$ and $150 \mathrm{~s}$ but then drops by four orders of magnitude as $\tau$ increases to $24 \mathrm{~h}$. The wave frequency spectra for $k_{v}^{\prime}=1$ [Fig. 7(b)] and $k_{v}^{\prime}=2$ [Fig. 7(c)] have distinct peaks: a large one at the linear frequency $\omega_{L I N}$ and a much smaller one at the buoyancy frequency $N$. The peaks at the linear frequency have roughly equal amplitudes for $\tau \leq 1 \mathrm{~h}$ and then decrease and disappear for larger $\tau$. Otherwise the dependence on $\tau$ is similar to the geostrophic spectra, with smaller $\tau$ yielding more energy at higher frequencies than larger $\tau$.

Forced geostrophic modes have $k_{h}^{\prime} \approx 4$ and $k_{v}^{\prime}=1$. Wave vectors of modes directly interacting with two forced geostrophic modes will therefore have horizontal component $\$ 8$ and vertical component 0 or 2 . In fact, most of the transfer from geostrophic to wave energy by WGG interactions happens at $k_{v}^{\prime}=2$ (Fig. 8). Consider, for example, wave modes with $k_{h}^{\prime} \approx 4$ and $k_{v}^{\prime}=2$, the frequency spectra of which are shown in Fig. 7(c). The dimensionless linear frequency of such modes is $\omega^{\prime} \approx 80$. It is therefore interesting to look at the energy in the forced geostrophic modes [Fig. 7(a)] around $\omega^{\prime}=80$. Figure 9 plots the total energy of these modes in the band $40 \leq \omega^{\prime} \leq 160$. The shape of this curve is remarkably similar to the dependence of wave energy on $\tau$ in Fig. 4. For $\tau \lesssim 1800 \mathrm{~s}$, the geostrophic energy at these frequencies is roughly independent of $\tau$. For these cases, the forcing time scale is sufficiently short that the excitation of this band is insensitive to $\tau$. For larger $\tau$, the energy in this frequency band falls off with increasing $\tau$. This rapid decrease with

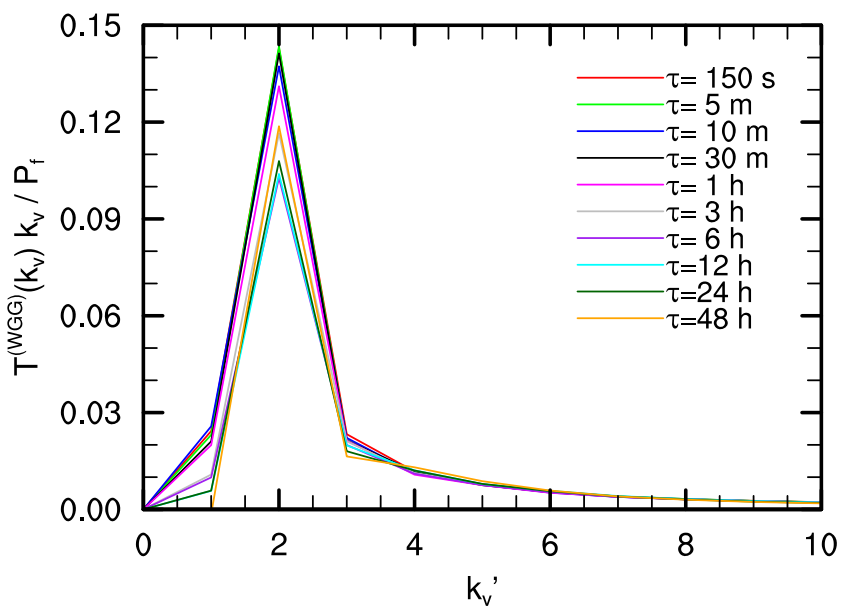

FIG. 8. Time-averaged $k_{v}$ wave energy transfer spectra due to WGG interactions for all $\tau$. Spectra are nondimensionalized by $P_{f}$. Linear-linear axes are used so that the transfer into $k_{v}=0$ can be seen. Only the range $0 \leq k_{v}^{\prime} \leq 10$ is shown, to clearly illustrate the peak at $k_{v}^{\prime}=2$. The transfer outside of this range is negligible.

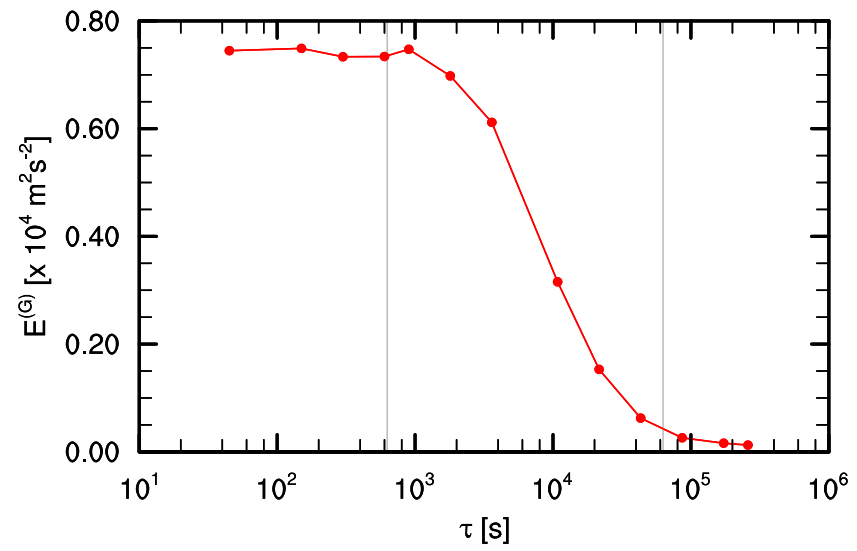

FIG. 9. Total geostrophic energy in the frequency band $40 \leq \omega^{\prime} \leq 160$ for simulations with different $\tau$. The vertical lines mark the buoyancy and inertial periods. 
increasing $\tau$ is due to the steepening of the geostrophic frequency spectrum, as expected for red noise forcing. The slope of the spectrum decreases from -2 for $\tau=45 \mathrm{~s}$ to -4 for $\tau=$ 24 h. The similar transition with $\tau$ in Figs. 4 and 9 supports the idea that forcing-induced oscillations in the geostrophic modes around the linear wave frequency enhance the transfer of geostrophic to wave energy.

\section{B. Dependence on resolution}

The vertical resolution employed above is relatively high, especially when compared to more realistic atmospheric models. ${ }^{7}$ However, the horizontal resolution is modest, and so we have checked the sensitivity of our results to increasing $n_{h}$. Rather than repeating the full set of $\tau$ values, we have focused on two extreme cases of small $\tau=150 \mathrm{~s}$ and large $\tau=24 \mathrm{~h}$. Simulations with both $\tau$ values were repeated with $n_{h}=512$ $(\Delta x=11.8 \mathrm{~km})$, and the small $\tau$ case was also repeated with $n_{h}=1024(\Delta x=5.9 \mathrm{~km})$. The resulting levels of wave energy are included in Fig. 4. The wave energy decreases slightly with increasing resolution, although the mean values are still well within the error bars of the $n_{h}=256$ simulations. These simulations show that the overall trend found above, of more wave energy generated when $\tau$ is short, is not an artifact of low resolution.
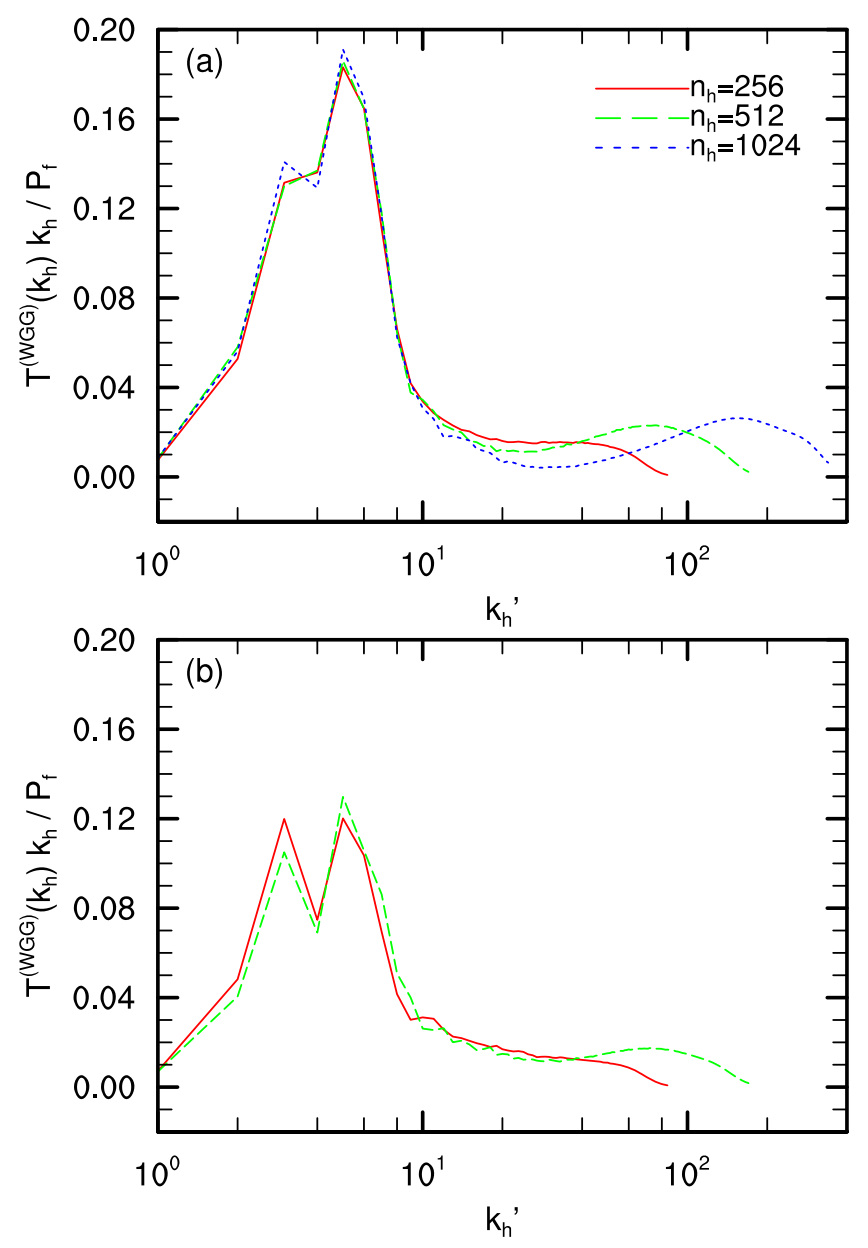

FIG. 10. Wave energy transfer spectra due to WGG interactions for (a) $\tau=150 \mathrm{~s}$ and (b) $24 \mathrm{~h}$ at different horizontal resolutions. Other details are as in Fig. 5.
The reason for the insensitivity to resolution is that, despite the modest resolution, the forcing and resulting conversion of geostrophic to wave energy are large-scale and therefore well resolved. Figure 10 shows the WGG transfer spectra for $\tau=150 \mathrm{~s}$ and $24 \mathrm{~h}$ for different resolutions. The dependence of the transfer spectra on resolution at large scales, where most of the conversion from geostrophic to wave energy occurs, is small.

\section{Dependence on $\boldsymbol{N}$ and $f$}

To investigate the possible dependence of these results on $N$ and $f$, and hence the linear wave frequencies, we consider a set of simulations with $N$ and $f$ twice as large as above. Doubling both $N$ and $f$ is convenient because the linear frequencies at all $\boldsymbol{k}$ will also double. The forcing-based Rossby and Froude numbers are correspondingly reduced by a factor of two. The resulting KE-based velocity scale is around $10.5 \mathrm{~ms}^{-1}$ for all $\tau$, which is only slightly larger than what was obtained with smaller $N$ and $f$. The resulting levels of wave energy are reduced at all $\tau$, likely because of the weaker offresonant interactions due to the smaller $\mathrm{Ro}$ and $\mathrm{Fr}$ in these simulations. For example, for $\tau=45 \mathrm{~s},\left\langle E^{(W)} / E\right\rangle=0.069$ compared to 0.19 for the smaller $N$ and $f$. The wave energy fractions $\left\langle E^{(W)} / E\right\rangle$ are plotted in Fig. 11. To facilitate comparison with the above simulations, the ratios for both sets
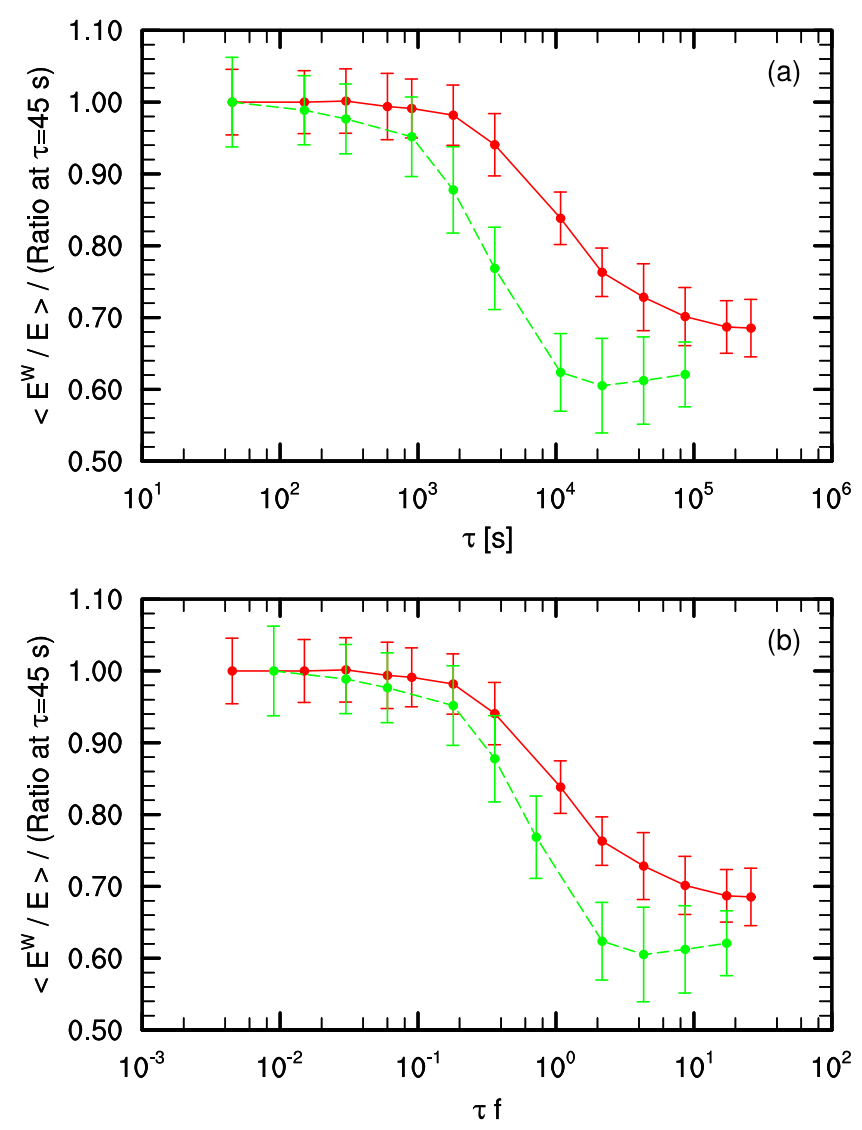

FIG. 11. Time-averaged ratio of wave energy to total energy for simulations with different $\tau$. Colors correspond to runs with standard (red, solid) and double (green, dashed) values of $N$ and $f$. Ratios are normalized by their respective values at $\tau=45 \mathrm{~s}$ and are plotted against (a) $\tau$ and (b) $\tau f$. Other details are as in Fig. 4. 
of simulations are scaled by their respective $\tau=45 \mathrm{~s}$ values. Given the importance of the relationship between $\tau$ and the linear frequencies suggested by the above results, the ratios in Fig. 11 are plotted against $\tau$ and $\tau f$. Since $N$ and $f$ are both doubled in these tests, the linear frequencies of all modes vary like $f$.

As mentioned earlier, there is a transition from larger to smaller levels of wave energy as $\tau$ increases. However, with larger $N$ and $f$, this transition happens at smaller values of $\tau$ [Fig. 11(a)]. Plotting the wave energy ratio against $\tau f$ [Fig. 11(b)] collapses some of this difference, and the drop in wave energy with increasing $\tau$ is found to occur around $\tau f \gtrsim 0.2$ in both cases. However, this drop begins at slightly smaller $\tau f$ and is more rapid for the larger $N$ and $f$ simulations. Since these simulations have weaker off-resonant interactions, they may be more sensitive to $\tau$. While there appears to be a local minimum in wave energy at $\tau f \approx 4$ in the larger $N$ and $f$ simulations, given the error bars, both sets of simulations are consistent with the wave energy approaching a constant level for $\tau f \gtrsim 10$.

Frequency spectra for simulations with the small and large values of $N$ and $f$ are compared in Fig. 12. For clarity, only $\tau=30 \mathrm{~min}$ and $1 \mathrm{~h}$ are shown, both of which show a strong dependence on $N$ and $f$ in Fig. 11(a). In addition,
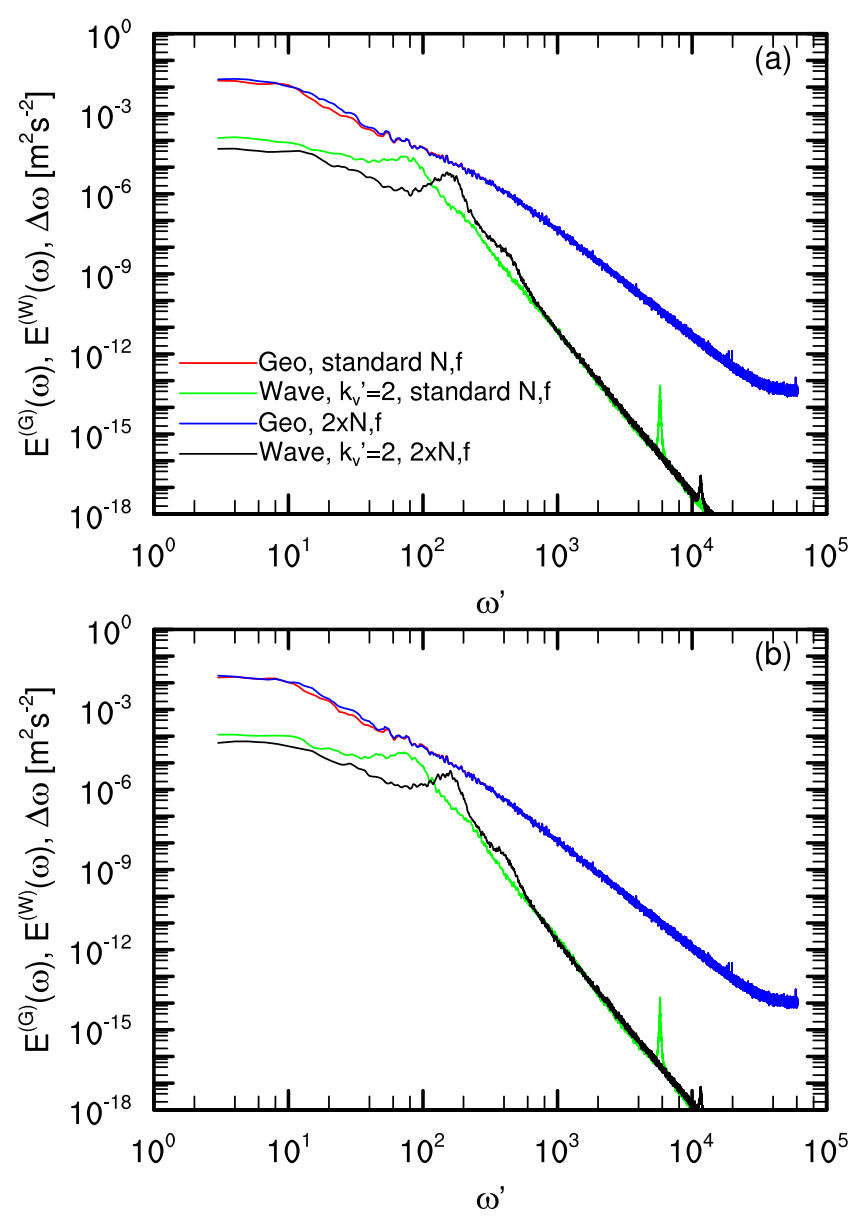

FIG. 12. Average frequency spectra of geostrophic modes for nine wave vectors with $k_{h}^{\prime} \approx 4$ and $k_{v}^{\prime}=1$ and wave modes for nine wave vectors with $k_{h}^{\prime} \approx 4$ and $k_{v}^{\prime}=2$. Simulations with standard and double values of $N$ and $f$ are shown at (a) $\tau=30 \mathrm{~min}$ and (b) $\tau=1 \mathrm{~h}$. Other details are as in Fig. 7 . only geostrophic modes in the forcing band and wave modes with $k_{v}^{\prime}=2$ are considered. For both $\tau$ values, the frequency spectra of geostrophic energy are relatively independent of $N$ and $f$. By contrast, the wave spectra are quite different, since they are peaked at the linear wave frequency, which is twice as large for the simulations with larger $N$ and $f$. In all cases, the size of the peak in the wave spectrum at the linear frequency generally follows the amplitude of the geostrophic spectrum at the same frequency. Since there is less geostrophic energy at the linear frequency in the large $N$ and $f$ simulations, the wave energy peak is correspondingly smaller.

\section{CONCLUSIONS}

We have presented simulations of rotating stratified turbulence with red noise forcing of large-scale geostrophic modes. Simulations are designed as an idealization of the atmospheric mesoscale. The decorrelation time scale $\tau$ of the noise is varied from $3 \Delta t$ (i.e., nearly white noise) to the slower time scale of the forced vortices. Wave modes are not forced directly. Nevertheless, the conversion of geostrophic energy into waves, and the resulting level of wave energy, is found to vary with $\tau$. Short $\tau$ values yield greater transfer into wave energy and about $46 \%$ more wave energy than larger $\tau$. The drop in wave energy occurs as $\tau f$ exceeds 0.2 , although it is more rapid for the case with larger $N$ and $f$, possibly due to weaker off-resonant interactions when $R o$ is smaller. Since the conversion of geostrophic to wave energy occurs at large scales, these findings are insensitive to increases in grid resolution.

The dependence on $\tau$ has been explored by examining the frequency spectra of geostrophic and wave modes at various wave vectors. Random forcing excites a range of frequencies in the forced geostrophic modes. The value of $\tau$ controls the amount of energy in the high frequencies. When $\tau$ is short, the resulting frequency spectrum is broad, and there is significant energy at higher frequencies. When $\tau$ is longer, the spectrum steepens and the high-frequency energy is reduced. We argue that geostrophic-wave transfer is enhanced when wave modes are excited, through nonlinear interactions, around their linear frequency. Such interactions happen more readily when the forced geostrophic modes have more energy at the linear frequency of the waves with which they interact. This hypothesis is supported by frequency spectra of the interacting wave modes, which are peaked at their linear frequency. The size of this peak follows the geostrophic energy spectrum. When the geostrophic energy at the linear frequency is increased-e.g., by decreasing $\tau$ or decreasing the linear frequency through $N$ and $f$-the transfer to the waves is stronger. By contrast, when the geostrophic energy at the linear frequency is decreased-e.g., by increasing $\tau$ or increasing the linear frequency - the transfer to the waves is reduced.

As an ad hoc model for the excitation of large-scale quasigeostrophic vortices, white noise forcing is simple but not very realistic. Large-scale vortices are generated in nature by baroclinic instability or nonlinear interactions with other 
vortices. These processes occur on the time scale of the largescale vortices. White noise forcing excites unnaturally high frequencies in the geostrophic motion. The energy in these frequencies is not benign; it yields an unrealistically large transfer to wave energy and almost $50 \%$ more wave energy at small $\tau$ compared to large. This effect is not enormous and might be acceptable in simulations where the generation of waves is not of particular interest. However, in studies where the level of wave energy is critical-e.g., for the transition of the energy spectrum from a -3 to $-5 / 3$ slope s $^{3,14,15}$-it is probably safest to avoid white noise forcing and use larger $\tau$ closer to the time scale of the forced vortices or another forcing approach altogether. $^{4,7}$

\section{ACKNOWLEDGMENTS}

This paper benefited greatly from comments by three anonymous reviewers. Some simulations were performed on the facilities of the Shared Hierarchical Academic Research Computing Network (SHARCNET: www.sharcnet.ca) and Compute/Calcul Canada. Financial support from the Natural Sciences and Engineering Research Council of Canada (Grant No. RGPIN-386456-2015) is gratefully acknowledged.

${ }^{1}$ K. Alvelius, "Random forcing of three-dimensional homogeneous turbulence," Phys. Fluids 11, 1880-1889 (1999).

${ }^{2}$ J. Sukhatme and L. M. Smith, "Vortical and wave modes in 3D rotating stratified flows: Random large-scale forcing," Geophys. Astrophys. Fluid Dyn. 102, 437-455 (2008).

${ }^{3}$ E. Deusebio, A. Vallgren, and E. Lindborg, "The route to dissipation in strongly stratified and rotating flows," J. Fluid Mech. 720, 66-103 (2013).

${ }^{4}$ N. P. Sullivan, S. Mahalingam, and R. M. Kerr, "Deterministic forcing of homogeneous, isotropic turbulence," Phys. Fluids 6, 1612-1614 (1994).

${ }^{5}$ L. Bourouiba, D. N. Straub, and M. L. Waite, "Non-local energy transfers in rotating turbulence at intermediate Rossby number," J. Fluid Mech. 690, 129-147 (2012).

${ }^{6} \mathrm{D}$. Chung and G. Matheou, "Direct numerical simulation of stationary homogeneous stratified sheared turbulence," J. Fluid Mech. 696, 434-467 (2012).

${ }^{7}$ M. L. Waite, "Dependence of model energy spectra on vertical resolution," Mon. Weather Rev. 144, 1407-1421 (2016).

${ }^{8}$ L. Biferale, A. S. Lanotte, and F. Toschi, "Effects of forcing in threedimensional turbulent flows,” Phys. Rev. Lett. 92, 094503 (2004).
${ }^{9} \mathrm{P}$. Bartello, "Geostrophic adjustment and inverse cascades in rotating stratified turbulence," J. Atmos. Sci. 52, 4410-4428 (1995).

${ }^{10}$ Y. Kitamura and Y. Matsuda, "Energy cascade processes in rotating stratified turbulence with application to the atmospheric mesoscale," J. Geophys. Res. 115, D11104, https://doi.org/10.1029/2009jd012368 (2010).

${ }^{11}$ S. Kurien and L. M. Smith, "Effect of rotation and domain aspect-ratio on layer formation in strongly stratified Boussinesq flows," J. Turbul. 15, 241-271 (2014).

${ }^{12}$ C. Herbert, R. Marino, D. Rosenberg, and A. Pouquet, "Waves and vortices in the inverse cascade regime of stratified turbulence with or without rotation," J. Fluid Mech. 806, 165-204 (2016).

${ }^{13}$ M. L. Waite and P. Bartello, "The transition from geostrophic to stratified turbulence," J. Fluid Mech. 568, 89-108 (2006).

${ }^{14}$ Y. Kitamura and Y. Matsuda, "The $\mathrm{k}_{\mathrm{h}}{ }^{-3}$ and $\mathrm{k}_{\mathrm{h}}{ }^{-5 / 3}$ energy spectra in stratified turbulence," Geophys. Res. Lett. 33, L05809, https://doi.org/ 10.1029/2005gl024996 (2006).

${ }^{15}$ P. Bartello, "Quasigeostrophic and stratified turbulence in the atmosphere," in IUTAN Symposium on Turbulence in the Atmosphere and Oceans, IUTAM Bookseries Vol 28, edited by D. G. Dritschel (Springer, 2010), pp. 117-130.

${ }^{16}$ M. L. Waite and P. Bartello, "Stratified turbulence generated by internal gravity waves," J. Fluid Mech. 546, 313-339 (2006).

${ }^{17}$ G. D. Nastrom and K. S. Gage, "A climatology of atmospheric wavenumber spectra observed by commercial aircraft," J. Atmos. Sci. 42, 950-960 (1985).

${ }^{18}$ A. Babin, A. Mahalov, B. Nicolaenko, and Y. Zhou, "On the asymptotic regimes and the strongly stratified limit of rotating Boussinesq equations," Theor. Comput. Fluid Dyn. 9, 223-251 (1997).

${ }^{19}$ P. F. Embid and A. J. Majda, "Low Froude number limiting dynamics for stably stratified flow with small or finite Rossby numbers," Geophys. Astrophys. Fluid Dyn. 87, 1-50 (1998).

${ }^{20}$ L. M. Smith and F. Waleffe, "Generation of slow large scales in forced rotating stratified turbulence," J. Fluid Mech. 451, 145-168 (2002).

${ }^{21}$ H. A. Kafiabad and P. Bartello, "Balance dynamics in rotating stratified turbulence,” J. Fluid Mech. 795, 914-949 (2016).

${ }^{22}$ M.-P. Lelong and J. J. Riley, "Internal wave-vortical mode interactions in strongly stratified flows," J. Fluid Mech. 232, 1-19 (1991).

${ }^{23}$ D. R. Durran, Numerical Methods for Fluid Dynamics (Springer, 2010).

${ }^{24}$ M. Frigo and S. Johnson, "The design and implementation of FFTW3," Proc. IEEE 93, 216-231 (2005).

${ }^{25}$ S. A. Orszag, "Numerical simulation of incompressible flows with simple boundaries. I. Galerkin (spectral) representations," Stud. Appl. Math. 50, 293-327 (1971).

${ }^{26} \mathrm{~J}$. R. Herring and O. Métais, "Numerical experiments in forced stably stratified turbulence,” J. Fluid Mech. 202, 97-115 (1989).

${ }^{27}$ Y. Kimura and J. R. Herring, "Energy spectra of stably stratified turbulence," J. Fluid Mech. 698, 19-50 (2012).

${ }^{28}$ K. Hamilton, Y. O. Takahashi, and W. Ohfuchi, "The mesoscale spectrum of atmospheric motions investigated in a very fine resolution global general circulation model," J. Geophys. Res. 113, D18110, https://doi.org/ 10.1029/2008jd010115 (2008). 